\title{
Urinary megalin in association with progression factors of diabetic nephropathy
}

\author{
Akour $\mathrm{A}^{1}$, Kasabri $\mathrm{V}^{1}$, Bulatova $\mathrm{N}^{1}$, Al-Motassem $\mathrm{Y}^{1}$, Fahmawi $\mathrm{H}^{1}$, Momani $\mathrm{M}^{2}$, Zayed $\mathrm{A}^{2}$, \\ Alquoqa $\mathrm{R}^{1}$, AlHawari $\mathrm{H}^{3}$
}

Department of Biopharmaceutics and Clinical Pharmacy, School of Pharmacy, the University of Jordan, Amman, Jordan. a.akour@ju.edu.jo

\begin{abstract}
AIM: The aim of this study is to evaluate the association between urinary megalin, renal function, blood pressure, lipid profile, vitamin $D$ and glycemic control in patients with type 2 diabetes mellitus (T2DM).

METHODS:. This was a cross-sectional study which recruited 209 patients with T2DM. Urinary megalin was positively associated with systolic blood pressure (SBP) $(r=0.218, p=0.04)$ but negatively with glomerular filtration rate (GFR) $(r=-0.16, p=0.023)$. The levels of urinary albumin, triglycerides (TGs) and glycosylated hemoglobin (HbA1c) were higher in the "high-megalin" group, compared to those in "low-megalin" group. Moreover, there was a significant inverse association between vitamin $D_{3}$ levels and megalin levels in urine $(\mathrm{OR}=0.281, \mathrm{p}=0.047)$.

CONCLUSION: Our study showed for the first time that megalin is associated with progression factors of diabetic nephropathy as well as vitamin D deficiency (Tab. 3, Fig. 1, Ref. 15). Text in PDF www.elis.sk.

KEY WORDS: diabetic nephropathy, progression factors, biomarker, type 2 diabetes, megalin.
\end{abstract}

\section{Introduction}

Urinary albumin excretion has been used as an established diagnostic and progression marker for diabetic nephropathy (DN). $(1,2)$ Recently, urinary megalin excretion has been evaluated as a potential urinary marker of nephropathy( 3 ). In fact, urinary fulllength megalin was correlated with the severity of DN in patients with T2DM and its levels were significantly high even in normoalbuminuric patients (3). However, the possibility to use megalin as a marker for predicting therapeutic effects/interventions on the progression of diabetic nephropathy has not been studied. Therefore, this study is aimed at evaluating the association between urinary megalin, renal function, blood pressure, lipid profile, vitamin D and glycemic control in patients with T2DM who have any degree of chronic kidney disease (CKD) and/or albuminuria. This study will enable us to provide further validation of megalin as a urinary marker for DN as well as possibility to predict the development and/or progression of diabetic nephropathy (Fig. 1).

${ }^{1}$ Department of Biopharmaceutics and Clinical Pharmacy, School of Pharmacy, the University of Jordan, Amman, Jordan, ${ }^{2}$ Diabetes and Endocrinology Unit, Jordan University Hospital, Amman, Jordan, and ${ }^{3}$ Nephrology Unit, Jordan University Hospital, Amman, Jordan

Address for correspondence: A. Akour. Department of Biopharmaceutics and Clinical Pharmacy, School of Pharmacy, the University of Jordan, Amman, Jordan.

Acknowledgments: The authors would like to thank the Deanship of Academic Research and Quality Assurance at the University of Jordan and the Scientific Research Fund at the Ministry of Higher Education for the funding.

\section{Methods}

Settings

This was a cross-sectional study that recruited patients with diagnosed T2DM (without attention to the duration of the diagnosis), or any degree of CKD and/or albuminuria who were treated at the Jordan University Hospital (JUH) in the period between September 2014 and September 2017. The study was approved by the JUH Institutional Review Board (IRB), while all patients and/or their guardians provided written informed consent to participate in the study. To enhance patients' participation, vitamin D supplements were provided to each patient.

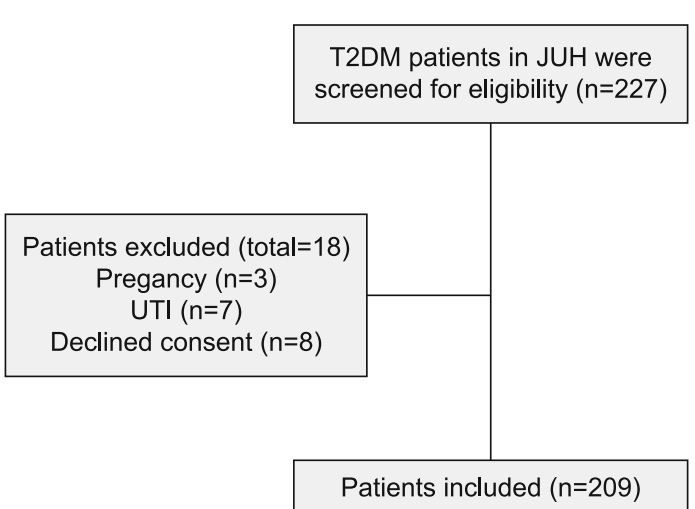

Fig. 1. T2DM: type 2 diabetes mellitus, JUH: Jordan University Hospital, UTI: urinary tract infection. 
Tab. 1. Descriptive summary of patients' clinical parameters $(n=209)$.

\begin{tabular}{|c|c|c|c|c|c|}
\hline & \multirow{2}{*}{ Mean } & \multirow{2}{*}{ Median } & \multirow{2}{*}{$\mathrm{SD}$} & \multicolumn{2}{|c|}{ Percentiles } \\
\hline & & & & 25 & 75 \\
\hline Age (years) & 55.56 & 56.50 & 10.065 & 48.25 & 63.75 \\
\hline BMI $\left(\mathrm{kg} / \mathrm{m}^{2}\right)$ & 31.68 & 31.02 & 7.41 & 27.43 & 35.16 \\
\hline $\mathrm{DBP}(\mathrm{mm} \mathrm{Hg})$ & 80.03 & 80.00 & 10.33 & 75.00 & 88.00 \\
\hline $\mathrm{SBP}(\mathrm{mm} \mathrm{Hg})$ & 131.4 & 130.0 & 16.99 & 120.0 & 140.0 \\
\hline Waist Circumference $(\mathrm{cm})$ & 112.10 & 111.00 & 11.449 & 106.50 & 117.00 \\
\hline GFR (mL/min) & 106.51 & 96.55 & 55.03 & 62.795 & 141.21 \\
\hline $\mathrm{HbA} 1 \mathrm{c} \%$ & 9.12 & 7.35 & 13.28 & 6.52 & 8.87 \\
\hline $\mathrm{FPG}(\mathrm{mg} / \mathrm{dL})$ & 170.61 & 156.45 & 72.97 & 115.1 & 204.0 \\
\hline BUN ( mg/dL) & 34.11 & 29.00 & 28.428 & 22.13 & 36.90 \\
\hline Vitamin $\mathrm{D}_{3}(\mathrm{ng} / \mathrm{dL})$ & 26.12 & 23.75 & 13.98 & 15.30 & 37.40 \\
\hline Total cholesterol (mg/dL) & 180.01 & 147.0 & 182.37 & 130.0 & 184.0 \\
\hline $\mathrm{LDL}-\mathrm{C}(\mathrm{mg} / \mathrm{dL})$ & 178.3 & 96.0 & 253.5 & 75.5 & 145.0 \\
\hline HDL-C (mgldL) & 43.74 & 42.0 & 16.31 & 33.0 & 51.0 \\
\hline Calcium (mg/dL) & 10.02 & 9.47 & 4.12 & 9.10 & 9.70 \\
\hline Phosphate (mg/dL) & 3.63 & 3.40 & 1.25 & 2.96 & 3.87 \\
\hline PTH $(\mathrm{pg} / \mathrm{ml})$ & 109.08 & 83.90 & 138.78 & 31.40 & 128.25 \\
\hline Megalin (pmol/g creatinine) & 155.05 & 110.0 & 128.04 & 74.0 & 171.00 \\
\hline
\end{tabular}

\section{Participants and Data collection}

Patients were included if they met the following criteria: (1) Adult female or male patients who are 18 years old or older, (2) Patients with the history of T2DM with no or any degree of CKD and/or albuminuria. Patients were excluded in cases of pregnancy or breast-feeding, or other conditions that might cause albuminuria (e.g., urinary tract infection, glomerulonephritis or other causes of glomerulonephropathies), and systemic diseases that might involve the kidneys (systemic lupus erythematosus, Sjögren's syndrome,

Tab. 2. Correlation between urinary megalin ( $\mathrm{pmol} / \mathrm{g}$ of creatinine) and clinical parameters.

\begin{tabular}{lcc}
\hline Clinical parameter & Correlation coefficient & P-value \\
\hline BMI & -0.171 & 0.013 \\
SBP & 0.218 & 0.041 \\
GFR & -0.157 & 0.023 \\
Calcium & -0.199 & 0.004 \\
\hline
\end{tabular}

BMI - body mass index; SBP - systolic blood pressure, GFR - glomerular filtration rate vasculitis, sarcoidosis, myeloma, HIV, syphilis, amyloidosis, congenital nephrotic syndrome).

Sample collection and determination of megalin urine levels

The method of S. Ogasawara was used with modifications (3). Urine was centrifuged and the pellet was assayed using the human megalin detection kit (Cusabio Technology Llc, USA). Megalin excretion rate was calculated in $\mathrm{pmol} / \mathrm{g}$ of creatinine. Serum creatinine and vitamin $\mathrm{D}_{3}$ levels, urinary creatinine, and urinary albumin were obtained from patients' data files.

\section{Sample size}

Sample size was calculated by using $\mathrm{G}^{*}$ power 3.0.10, according to Ogasawara et al (3), to detect $r^{2}$ of 0.76 between megalin levels and eGFR; sample size of 200 patients is required to achieve a power of 0.8 and $\alpha=0.05$

Statistical analysis

Patients' baseline variables were summarized as mean \pm standard deviation and median with interquartile range. Indepen-

Tab. 3. Comparison of kidney function, vitamin D3 and FPG per megalin group.

\begin{tabular}{|c|c|c|c|c|c|}
\hline & \multicolumn{2}{|c|}{ High megalin $(n=60)$} & \multicolumn{2}{|c|}{ Low megalin $(n=149)$} & \multirow{2}{*}{$\mathrm{p}$-Value } \\
\hline & Mean & $\mathrm{SD}$ & Mean & $\mathrm{SD}$ & \\
\hline$\overline{\mathrm{HbA} 1 \mathrm{c}}$ & 9.68 & 3.52 & 7.70 & 1.51 & 0.032 \\
\hline FPG & 170 & 73.1 & 170 & 59.8 & NS \\
\hline SBP & 130 & 4.60 & 131 & 7.06 & NS \\
\hline DBP & 79.8 & 2.92 & 80.1 & 4.26 & NS \\
\hline LDL-C & 113 & 62.0 & 110 & 55.8 & NS \\
\hline HDL-C & 45.1 & 13.9 & 43.1 & 12.0 & NS \\
\hline BMI & 30.4 & 6.48 & 32.1 & 5.67 & 0.016 \\
\hline GFR & 95.8 & 40.3 & 110 & 41.4 & 0.047 \\
\hline ACR & 37.1 & 12.1 & 33.8 & 7.71 & 0.031 \\
\hline
\end{tabular}

HbA1c - glycosylated hemoglobin, FPG - fasting plasma glucose, SBP - systolic blood pressure, DBP - diastolic blood pressure, TG - triglycerides LDL-C - low-density lipoprotein, HDL-C - high-density lipoprotein cholesterol, BMI - body mass index, GFR - glomerular filtration rate, ACR - albumin-to-creatinine ratio, NS - not significant 
dent student's t-test was used to compare clinical parameters of patients with high megalin and those with low megalin. Pearson's correlation test was used to assess the relationship between urinary megalin levels and various clinical parameters; then a stepwise regression was used to assess the association between serum levels of vitamin $\mathrm{D}_{3}$ and urinary megalin to adjust for various covariates. Statistical analysis was performed with SPSS 20 software.

\section{Results}

Two hundred and nine patients were included in the study. The description of clinical parameters is found in Table 1. Thereafter, we evaluated the correlation between urinary megalin levels and clinical parameters. Interestingly, pellet urinary megalin was positively associated with $\operatorname{SBP}(r=0.218, \mathrm{p}=0.04)$ but negatively correlated with GFR as a measure of kidney function ( $\mathrm{r}=-0.16$, $\mathrm{p}=0.023$ ). In addition, when patients were divided according to megalin cut-off point level that qualifies failure defined by Ogasawara et al (3) of urinary megalin $523.5 \mathrm{fmol} / \mathrm{g}$ creatinine (which is equivalent to $62.8 \mathrm{pg} / \mathrm{g}$ creatinine), to two groups with high pellet megalin and low megalin levels: urinary albumin, and TGs were higher in the "high-megalin" group compared to those in low-megalin group. HbAlc was statistically and significantly higher in the group with high urinary megalin (Tabs 2 and 3). A stepwise forward logistic regression model was performed to study the effect of vitamin D deficiency on megalin groups, while taking into account covariates as follows: SBP, FPG, and calcium levels. The results in Table 4 show that there is a significant negative association between the levels of vitamin D and urine megalin (OR $=0.281, \mathrm{p}=0.047 ; 95 \% \mathrm{CI}: 0.08-0.98)$, meaning that high level of deficiency in vitamin $\mathrm{D}$ will increase the probability of having high urinary megalin levels.

\section{Discussion and conclusions}

This is the first study to evaluate pellet urinary megalin levels in Jordanian T2DM patients and to correlate these levels with kidney functions and progression factors of renal diseases, as well as vitamin D status. In this study, urinary megalin was positively associated with $\mathrm{SBP}(\mathrm{r}=0.218, \mathrm{p}=0.04)$ but negatively correlated with GFR $(r=-0.16, p=0.023)$. In addition, when patients were divided according to urinary megalin cutoff point level (which qualifies as failure), urinary albumin, and TGs were higher in the "high-megalin" group, compared to those in "lowmegalin" group. HbA1c was statistically and significantly higher in the high-megalin group. Interestingly, this matches with the fact that aforementioned parameters (HbAlc, BP, and TGs), are associated with poorer progression of renal dysfunction. Moreover, there was a significant inverse association between vitamin $\mathrm{D}$ levels and urinary megalin levels $(\mathrm{OR}=0.281, \mathrm{p}=0.047)$. Megalin is involved in the reabsorption of various low molecular weight proteins that are filtered by glomeruli, such as albumin (4). Accordingly, megalin dysfunction may be implicated in the development of albuminuria in patients with diabetes mellitus, especially those with $\operatorname{T2DM}(5,6)$. In patients with type 1 diabetes mellitus, microalbuminuria is found to be associated with enhanced excretion of megalin and cubilin $(3,7,8)$ Ogasawara et al (3) assessed the clinical significance of different forms of megalin as novel biomarkers for diabetic nephropathy in patients with type 2 diabetes mellitus. They found that the urinary fulllength (C-) megalin was correlated with the severity of DN in patients with type 2 diabetes. Interestingly, C-megalin urine levels were significantly high in normoalbuminuric patients. Their elevation was in line with increased albuminuria and showed a better association with eGFR than with albuminuria. The upper normal cut-off points of urinary full-length megalin was determined in 160 normal controls as $523.5 \mathrm{fmol} / \mathrm{g}$ of urinary creatinine. (3) Based on the above, we can hypothesize that interventions that can affect megalin expression, could have an ameliorating effect on diabetic nephropathy.

In addition, the relationship between diabetic nephropathy and vitamin D deficiency has been studied (9-11). Similar to our study, the study of Diaz et al. (2009) (12) found a significant association between vitamin D deficiency/insufficiency and nephropathy in patients with diabetes mellitus. In addition, vitamin D deficiency $(<30 \mathrm{ng} / \mathrm{mL})$ was detected in $86 \%$ of CKD patients.(9) Moreover, in a cohort study by De Boer et al (2007), a strong association was found between the albuminuria rates and the decrease in vitamin D absorption (13). Although this may reflect the inability of a failing kidney to activate the vitamin D to calcitriol, it is also possible to explain this finding by the loss of megalin occurring in CKD patients.

Recently, a randomized controlled trial showed that the daily administration of $2 \mu \mathrm{g}$ paricalcitol, an analogue of the active form of vitamin D3, in patients receiving renin-angiotensin-aldosterone inhibtors, lowered the residual albuminuria in patients with diabetic nephropathy (14). A high dose of paricalcitol may have been needed to generate such a beneficial effect in patients with diabetic nephropathy because of the reduced renal functions of megalin in these patients. The mechanisms of paricalcitol action on the kidney remain unknown. Therefore, it would be of interest to investigate whether it acts on the renal megalin excretion. While the effect of paricalcitol add-on therapy on diabetic nephropathy was previously evaluated $(14,15)$ there is no study that would evaluate the association between vitamin D levels and diabetic nephropathy as measured by urinary megalin as a novel marker of DN.

The weakness of our study is inherent to cross-sectional character of our study, because rather than causation, it assesses merely the association. Many co-variates and medical intervention should be taken into consideration before we can confirm the true relationship.

Therefore, our study concluded that megalin is associated positively with progression factors of $\mathrm{DN}$ and negatively with vitamin $\mathrm{D}_{3}$ levels. In order to validate this protein as a prognostic marker, future studies should prospectively evaluate the change in megalin levels in patients with nephropathy, and assess the effect of interventions to slow down the progression of DN such as control of blood pressure, glycemic status, vitamin D, etc. on the levels or urinary megalin. 


\section{References}

1. Gross JL, de Azevedo MJ, Silveiro SP, Canani LH, Caramori ML, ZelmanovitzT. Diabetic nephropathy: diagnosis, prevention, and treatment. Diabetes Care 2005; 28 (1): 164-176.

2. Rheinberger M, Boger CA. (Diabetic nephropathy: new insights into diagnosis, prevention and treatment). Dtsch Med Wschr 2014; 139 (14): 704-706.

3. Ogasawara S, Hosojima M, Kaseda R, Kabasawa H, YamamotoKabasawa K, Kurosawa H, Sato H, Iino N, Takeda T, Suzuki Y, Narita I, Yamagata K, Tomino Y, Gejyo F, Hirayama Y, Sekine S, Saito A. Significance of urinary full-length and ectodomain forms of megalin in patients with type 2 diabetes. Diabetes Care 2012; 35 (5): $1112-1118$.

4. Akour AA, Kennedy MJ, Gerk P. Receptor-mediated endocytosis across human placenta: emphasis on megalin. Mol Pharm 2013; 10 (4): 1269-1278.

5. Saito A, Takeda T, Hama H, Oyama Y, Hosaka K, Tanuma A, Kaseda R, Ueno M, Nishi S, Ogasawara S, Gondaira F, Suzuki Y, Gejyo F. Role of megalin, a proximal tubular endocytic receptor, in the pathogenesis of diabetic and metabolic syndrome-related nephropathies: protein metabolic overload hypothesis. Nephrology (Carlton) 2005; 10 Suppl: S26-31

6. Oyama Y, Takeda T, Hama H, Tanuma A, Iino N, Sato K, Kaseda R, Ma M, Yamamoto T, Fujii H, Kazama JJ, Odani S, Terada Y, Mizuta K, Gejyo F, Saito A. Evidence for megalin-mediated proximal tubular uptake of L-FABP, a carrier of potentially nephrotoxic molecules. Lab Invest 2005; 85 (4): 522-531.

7. Thrailkill KM, Nimmo T, Bunn RC, Cockrell GE, Moreau CS, Mackintosh S, Edmondson RD, Fowlkes JL. Microalbuminuria in type 1 diabetes is associated with enhanced excretion of the endocytic multiligand receptors megalin and cubilin. Diabetes Care 2009; 32 (7): 1266-1268.
8. Fowlkes JL, Bunn RC, Cockrell GE, Clark LM, Wahl EC, Lumpkin CK, Thrailkill KM. Dysregulation of the intrarenal vitamin D endocytic pathway in a nephropathy-prone mouse model of type 1 diabetes. Exp Diabet Res 2011; 2011: 269378.

9. Gonzalez EA, Sachdeva A, Oliver DA, Martin KJ. Vitamin D insufficiency and deficiency in chronic kidney disease. A single center observational study. Am J Nephrol 2004; 24 (5): 503-510.

10. Del Valle E, Negri AL, Aguirre C, Fradinger E, Zanchetta JR. Prevalence of $25(\mathrm{OH})$ vitamin D insufficiency and deficiency in chronic kidney disease stage 5 patients on hemodialysis. Hemodial Int 2007; 11 (3): $315-21$

11. Diniz HF, Romao MF, Elias RM, Romao Junior JE. Vitamin D deficiency and insufficiency in patients with chronic kidney disease. J Bras Nefrol 2012; 34 (1): 58-63.

12. Diaz VA, Mainous, 3rd, AG, Carek PJ, Wessell AM, Everett CJ. The association of vitamin D deficiency and insufficiency with diabetic nephropathy: implications for health disparities. J Am Board Fam Med 2009; 22 (5): 521-527.

13. de Boer IH, Ioannou GN, Kestenbaum B, Brunzell JD, Weiss NS. 25-Hydroxyvitamin D levels and albuminuria in the Third National Health and Nutrition Examination Survey (NHANES III). Am J Kidney Dis 2007; 50 (1): 69-77.

14. de Zeeuw D, Agarwal R, Amdahl M, Audhya P, Coyne D, Garimella T, Parving HH, Pritchett Y, Remuzzi G, Ritz E, Andress D. Selective vitamin $\mathrm{D}$ receptor activation with paricalcitol for reduction of albuminuria in patients with type 2 diabetes (VITAL study): a randomised controlled trial. Lancet 2010; 376 (9752): 1543-1551.

15. Alborzi P, Patel NA, Peterson C, Bills JE, Bekele DM, Bunaye Z, Light RP, Agarwal R. Paricalcitol reduces albuminuria and inflammation in chronic kidney disease: a randomized double-blind pilot trial. Hypertension, 2008; 52 (2): 249-255. 